\title{
Kinetic-Thermodynamic Evidence for the Involvement of Set Steps in the Anionic Polymerization of Styrenes
}

Keywords: polymerization of styrenes • single electron transfer steps • kinetic-thermodynamic correlations

\section{INTRODUCTION}

It has been a common practice in the anionic polymerization of styrenes to employ an initiator of the benzylic type organolithium reagents generated by one of the methods $(1)-(3)^{1}$ :

$$
\begin{aligned}
\mathrm{BuLi}+\mathrm{Ph}_{2} \mathrm{C} & =\mathrm{CH}_{2} \rightarrow \mathrm{Ph}_{2} \mathrm{C}(\mathrm{Li}) \mathrm{CH}_{2} \mathrm{Bu} \\
\text { s-BuLi }+\mathrm{Ph}(\mathrm{Me}) \mathrm{C} & =\mathrm{CH}_{2} \rightarrow \mathrm{Ph}(\mathrm{Me})\left(\mathrm{CH}_{2} \mathrm{Bu}-\mathrm{s}\right) \mathrm{CLi} \\
2 \mathrm{Li}+2 \mathrm{Ph}_{2} \mathrm{C} & =\mathrm{CH}_{2} \rightarrow \mathrm{Ph}_{2} \mathrm{C}(\mathrm{Li}) \mathrm{CH}_{2} \mathrm{CH}_{2} \mathrm{C}(\mathrm{Li}) \mathrm{Ph}_{2}
\end{aligned}
$$

The reason appears to be that with benzyllic type organolithium reagents narrower distributions of molecular weights are obtained, which in turn implies that the latter type of organolithium reagents exhibit a greater reactivity toward styrenes than alkyllithium reagents. Indeed, the reactivity order of organolithiums appears to be dependent upon the reference substrate or the particular reaction which forms the basis for comparison. Thus, although butyllithium metalates toluene in the presence of the activating Lewis base tetrahydrofuran (THF), ${ }^{2}$ benzyllithium in the same solvent appears to be one order of magnitude more reactive than butyllithium toward triphenylmethane ${ }^{3}$ and 1,1-diphenylethylene. ${ }^{4}$ In fact, the relative rates for triphenylmethane metalation by the series $\mathrm{CH}_{3} \mathrm{Li}$, $\mathrm{PhLi}, \quad \mathrm{CH}_{2}=\mathrm{CHLi}, \quad \mathrm{CH}_{2}=\mathrm{CHCH}_{2} \mathrm{Li}, \quad n-\mathrm{BuLi}$, and $\mathrm{PhCH}_{2} \mathrm{Li}^{3}$ are well correlated with the ionization potentials ${ }^{5}$ of the free radical $\mathrm{R}^{\cdot}$ that corresponds to $\mathrm{RLi}^{6}$ (Fig. 1).

Both the metalation of triphenylmethane by organolithium reagents and the addition of the latter to 1,1-diphenylethylene lead to the formation of benzyllic type organolithiums and therefore are closely related reactions. Of particular relevance is the latter reaction because it serves as a prototype for the initiation and homopropagation steps of styrene polymerization. ${ }^{7}$

\section{RESULTS AND DISCUSSION}

We have noticed that benzylic type organolithiums such as $\mathrm{Ph}_{2}(\mathrm{Me}) \mathrm{CLi}, \mathrm{Ph}_{3} \mathrm{CLi}$, and $\mathrm{Ph}_{3} \mathrm{SnLi}$ on their reaction with aromatic ketones produce substantial amounts of the corresponding one-electron reduction products of the aromatic ketones. Namely, with benzophenone the yield of the corresponding ketyl was 14,11 , and $77 \%$ and with fluorenone $46,92.5$, and $93 \%$, respectively, ${ }^{8}$ e.g., eq. (4):

Journal of Polymer Science: Part A: Polymer Chemistry, Vol. 30, 1771-1773 (1992) (C) 1992 John Wiley \& Sons, Inc.

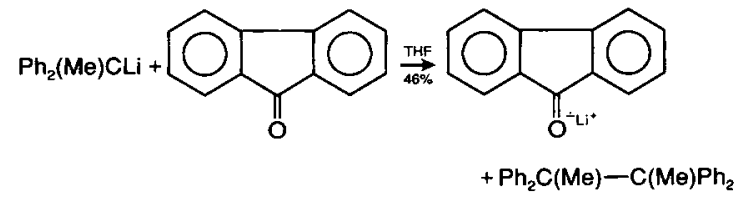

Given the close analogy between $\mathrm{Ph}_{2}(\mathrm{Me}) \mathrm{CLi}$ and the organometallic species on the right-hand side of eqs. (1$3)$, the ability of the latter to function as single electron donors appears to be very probable.

The reported specific rate constants for homopropagation of styrene in dioxane exhibit a marked dependence on the cation $\mathbf{M}^{+}$in the living polymer $-\mathbf{S}^{-} \mathbf{M}^{+}$. Namely, when $\mathrm{M}$ is varied as $\mathrm{Li}, \mathrm{Na}, \mathrm{K}, \mathrm{Rb}$, and $\mathrm{Cs}$, the $k_{p}$ 's are, respectively, ${ }^{9} 0.9,3.4,20,21.5$, and $24.5 M^{-1} \mathrm{~s}^{-1}$. This sequence seems to parallel the corresponding rate constants for the reaction of alkali metal benzophenone ketyls with $n$-propyl bromide in the same solvent, ${ }^{10}$ which are: $\mathrm{Li}, 0.0068 ; \mathrm{Na}, 3.4 ; \mathrm{K}, 63.8$; and $\mathrm{Cs}, 77.9 \mathrm{M}^{-1} \mathrm{~s}^{-1}$. In fact, the two sets of numbers plot reasonable linearly against each other (Fig. 2). This establishes then a close analogy between the two reactions, the second of which unequivocally involves single electron transfer (SET) steps. The corresponding $k_{p}$ 's in THF solvent are: $\mathrm{Li}, 160$; $\mathrm{Na}, 80 ; \mathrm{K}, 50, \mathrm{Rb}, 50$; and $\mathrm{Cs}, 22 .{ }^{11} \mathrm{With}$ the exception of the data for $\mathrm{Na}$, the rest of the data plot also linear against the corresponding specific rate constants for the reaction between alkali metal benzophenone ketyl and $n$-propyl bromide. The only difference is that the slope of the latter plot is of opposite sign, i.e., negative as compared to that of Figure 2. There is strong experimental evidence ${ }^{12}$ that the reactivity of radical anions exhibits a marked dependence on the counter-cation and that radical anions donate their single electron through the mediation of the cation. This mechanism suggests a relationship between the rate constants for electron transfer from a given anion and the donating ability of the cation. The latter is expressed by the ionization potential of $\mathbf{M}$ atom in the gas phase and it is defined by eq. (5):

$$
\operatorname{IP}(\mathrm{M})=\Delta H_{r}^{0}=\Delta H_{f}^{0}\left(\mathrm{M}^{+}\right)-\Delta H_{f}^{0}(\mathrm{M})
$$

Namely, IP (M) represents the enthalpy change in the gas phase and at $298.15 \mathrm{~K}$ of the reaction $\mathrm{M} \rightarrow \mathrm{M}^{+}+e^{-}$. In Figure 3 we plot $k_{p}$ 's against the respective IP (M)'s and as it can be seen there is a good linearity. The relationship in Figure 3 is of the Evans-Polanyi type, namely, it relates a kinetic parameter with a relevant thermodynamic one. ${ }^{13}$ Bearing in mind that Evans-Polanyi rela- 


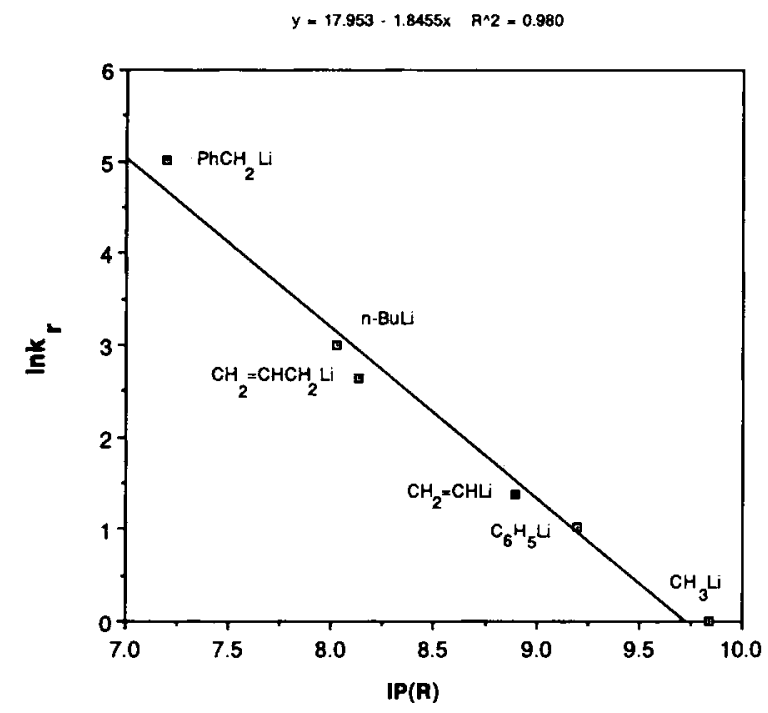

Figure 1. Logarithms of relative rate constants for triphenylmethane metalation by a sequence of RLi's in THF plotted against the gas phase ionization potential of the free radical that corresponds to $\mathrm{R}$ in $\mathrm{RLi}$. $k_{r}$ (at 0.01 formal concentration) from Ref. 3. IP ( $\left.\mathrm{R}^{*}\right)^{\prime}$ 's are: for $\mathrm{Ph}^{\circ}, \mathrm{I}$. P. Fisher, T. F. Palmer, and F. P. Lossing, J. Am. Chem. Soc., 86, 2741 (1964); for the rest, S. G. Lias, J. E. Bartmess, J. F. Liebman, R. D. Levin, and W. G. Mallard, "Gas Phase Ion and Neutral Thermochemistry," J. Phys. Chem. Ref. Data 17, (Supplement No. 1) (1988).

tionships hold, usually, for exoergic free radical reactions, ${ }^{13}$ we feel that relationship in Figure 3 is rather strong evidence for the involvement of SET steps in the anionic polymerization of styrenes. On the basis of the above information we propose the following mechanism, eq. (6):

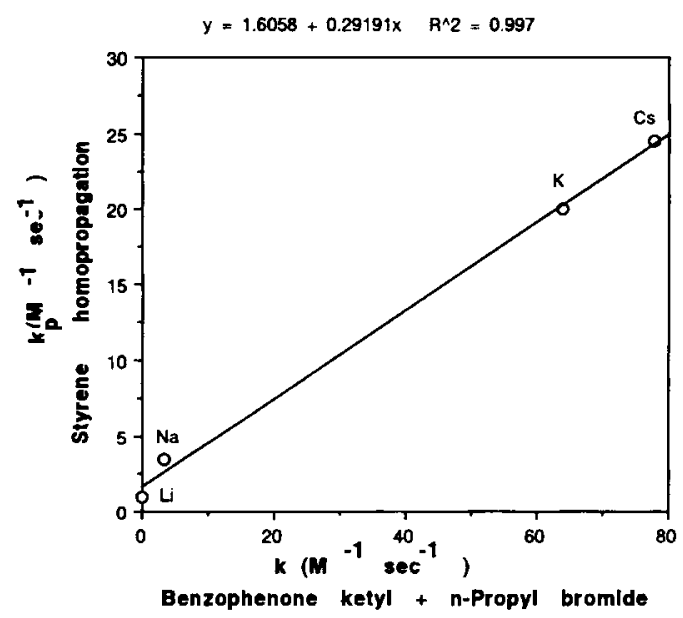

Figure 2. Specific rate constants for homopropagation of living polystyrene in dioxane plotted against the respective ones for the reaction of alkali metal benzophenone ketyls in dioxane with $n$-propyl bromide. Data from Refs. 9 and 10.

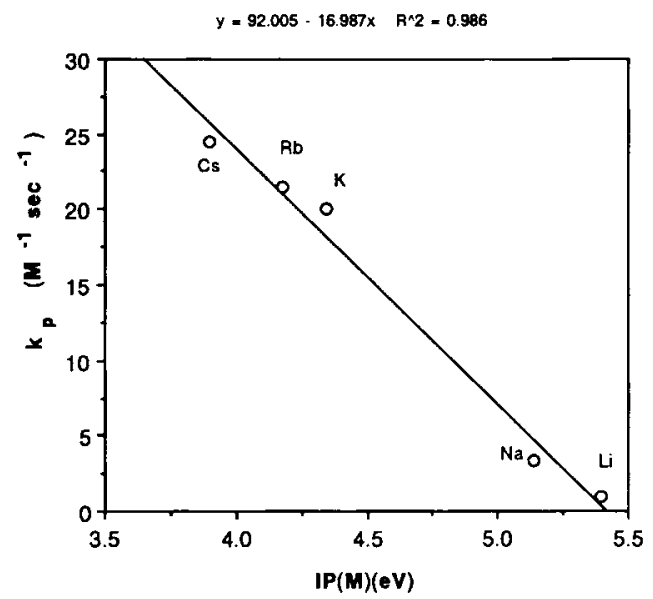

Figure 3. Specific rate constants for homopropagation of living polystyrene in dioxane plotted against the gas phase ionization potentials corresponding to the cation of the living polymer. $k_{p}$ 's (Dioxane) from Ref. 9; IP(M)'s from S. G. Lias, J. E. Bartmess, J. F. Liebman, R. D. Levin, and W. G. Mallard, "Gas Phase Ion and Neutral Thermochemistry," J. Phys. Chem. Ref. Data, 17 (Supplement No. 1) (1988).

$$
\begin{aligned}
&-\mathrm{S}^{-} \mathrm{M}^{+}+\mathrm{PhCH}=\mathrm{CH}_{2} \rightarrow\left(-\mathrm{S}^{*}, \mathrm{M}^{+},\left(\mathrm{PhCH}=\mathrm{CH}_{2}\right)^{*-} \leftrightarrow\right. \\
&\left.\mathrm{PhCH}-\mathrm{CH}_{2}^{+}\right) \rightarrow-\mathrm{SCH}_{2}(\mathrm{Ph}) \mathrm{CH}^{-} \mathrm{M}^{+}
\end{aligned}
$$

It should be noted that the inverse reactivity order that has been reported for the homopropagation step in styrene polymerization in $\mathrm{THF}^{11}$ solvent does not contradict the above mechanism. On the contrary, the $k_{p}$ 's (THF) exhibit also a linear dependence on IP (M) (Fig. 4), as do the corresponding $k_{p}$ 's (dioxane). The only difference is that the slopes of the straight lines in Figures 3 and 4 are of

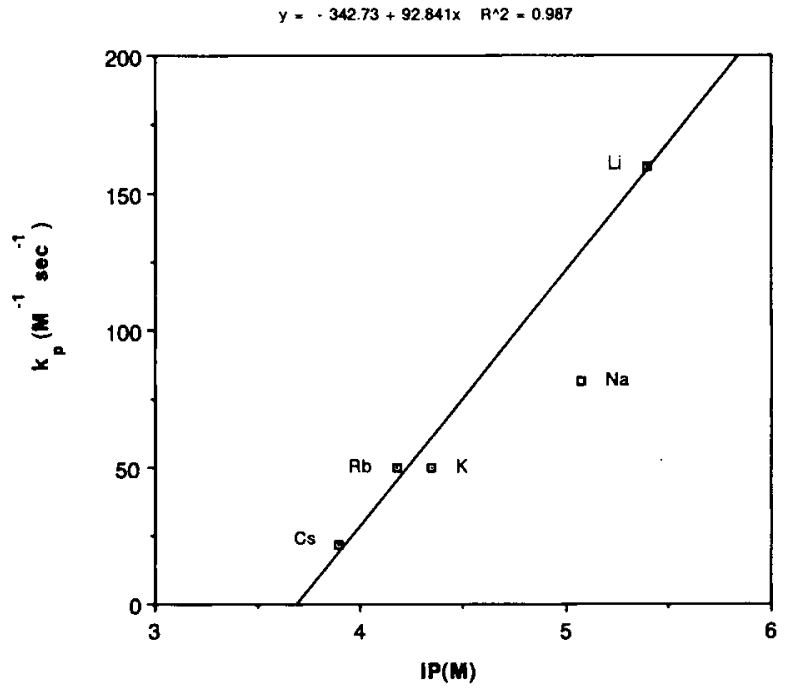

Figure 4. Specific rate constants for homopropagation of living polystyrene in THF plotted as in Fig. 3. $k_{p}$ (THF) from Ref. 11. 


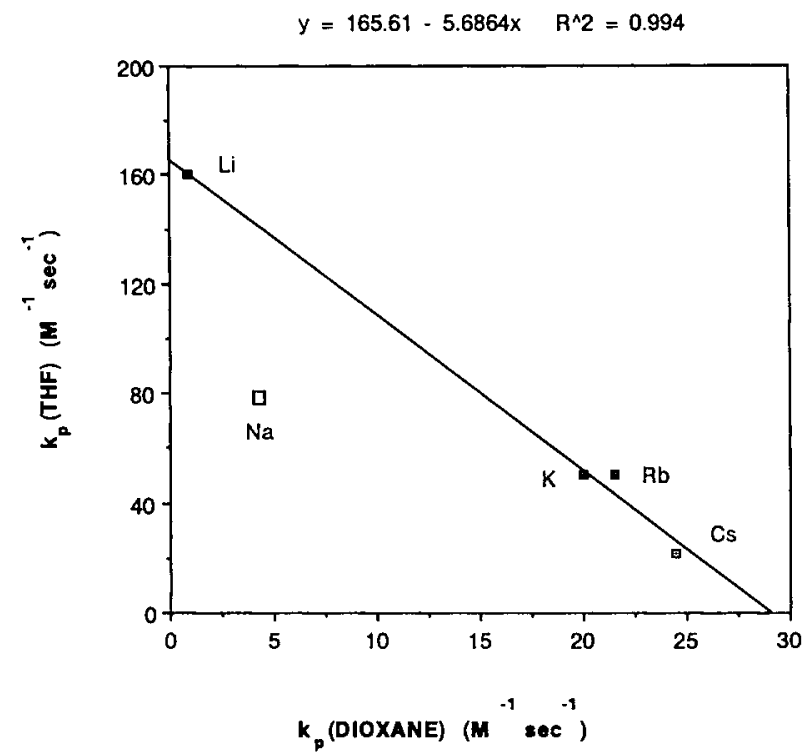

Figure 5. Specific rate constants for homopropagation of living polystyrene in THF plotted against the respective ones in dioxane.

opposite sign. Radical anions usually exhibit a reactivity order $\mathrm{Cs}>\mathrm{Rb}>\mathrm{K}>\mathrm{Na}>\mathrm{Li}$, but with certain substrates, such as an alkyl fluoride ${ }^{14}$ the reactivity order is inversed. This can be attributed to the fact that the reaction enthalpy, which is linearly related to a kinetic parameter, is governed by some factor of the reaction, e.g., enthalpies of formation versus enthalpies of solvation of the species involved in the reaction. Thus, the order $\mathrm{Li}>\mathrm{Na}>\mathrm{K}>$ $\mathrm{Rb}>\mathrm{Cs}$ that has been observed for the homopropagation of styrene in $\mathrm{THF}^{11}$ could be readily attributed to the enthalpy of solvation as being the predominant factor in the reaction enthalpy. This view is supported by the reported $^{11}$ correlation between $\log k_{p}(\mathrm{THF})$ and $1 /\left(r_{\mathrm{M}}+\right.$ 2).

As a corollary of the correlations in Figure 3 and 4 it follows that the two sets of $k_{p}$ 's in THF and dioxane should plot linearly against each other. This seems to be true for all $\mathrm{M}^{+}$'s but $\mathrm{Na}^{+}$(Fig. 5). Perhaps, the value for $\mathrm{Na}$ is in error. The estimated values for $k_{p}\left(\mathrm{Na}^{+}, \mathrm{THF}\right)$ by interpolation from the correlations in Figures 4 and 5 are 134 and $146 M^{-1} \mathrm{~s}^{-1}$, respectively.

\section{REFERENCES AND NOTES}

1. See, e.g., T. Ishizone, A. Hirao, and S. Nakahama, Macromolecules, 24, 625 (1991).

2. C. G. Screttas, J. F. Eastham, and C. W. Kamienski, Chimia, 24, 109 (1970).

3. P. West, R. Waack, and J. I. Purmort, J. Am. Chem. Soc., 92, 640 (1970).

4. R. Waack and M. A. Doran, J. Am. Chem. Soc., 91, 2456 (1969).
5. C. G. Screttas, J. Org. Chem., 44, 1471 (1979).

6. This successful correlation indicates that the comparison of the relative reactivities of the RLi's involved is sound. For an opposite view, see Ref. 4.

7. B. J. Wakefield, The Chemistry of Organolithium Compounds, Pergamon, Oxford, 1974, p. 92.

8. Accurately measured volumes of standard solutions of the organolithium and the ketone in THF were mixed under argon and the concentration of the ketyl produced was determined by measuring the paramagnetic solvent NMR shift: C. G. Screttas and M. MichaScrettas, J. Org. Chem., 46, 993 (1981).

9. M. Szwarc, Ion and Ion Pairs in Organic Reactions, Vol. 2, Wiley, New York, 1974, p. 378.

10. The specific rate constants are from H. V. Carter, B. J. McClelland, and E. Warhurst, Trans. Faraday Soc., 56, 343 (1960); A. Mathias and E. Warhurst, Trans. Faraday Soc., 58, 948 (1962) and were adjusted to a common substrate, i.e., $n$-propyl bromide. For example, the specific rate constant at $20^{\circ} \mathrm{C}$ for Csbenzophenone and $n$-propyl chloride is $0.20 \mathrm{M}^{-1} \mathrm{~s}^{-1}$. A ratio of the $\mathrm{k}(n-\mathrm{PrBr}) / k(n-\mathrm{PrCl})$ was calculated from the data for Na-phenylbiphenyl ketone. This ratio is $1.87 / 0.0048=389.6$. Assuming that this ratio depends not too strongly on the ketone and the cation we can apply it to the Cs-benzophenone; $k(n-\mathrm{PrBr})$ $=0.2 \times 389.6=77.9$. This assumption appears to be reasonable. Indeed, the enthalpies of formation of the pairs $\mathrm{NaCl}-\mathrm{NaBr}$ and $\mathrm{CsCl}-\mathrm{CsBr}$ differ by less than $2 \mathrm{kcal} / \mathrm{mol}$ and, therefore, its effect to the kinetics should be small also. For $\mathrm{K}$-benzophenone the $k_{20}(\mathrm{PhBr})=0.957 M^{-1} \mathrm{~s}^{-1}$. A ratio $k(n-\mathrm{PrBr}) /$ $k(\mathrm{PhBr})$ was obtained from the Na-benzophenone data, i.e., 66.67. Thus, $k(n-\mathrm{PrBr})$ for $K$-benzophenone $=66.67 \times 0.957=63.8 M^{-1} \mathrm{~s}^{-1}$.

11. D. N. Battacharyya, J. Smid, and M. Szwarc, J. Phys. Chem., 69, 624 (1965).

12. C. G. Screttas and M. Micha-Screttas, J. Phys. Chem., 87, 3844 (1983) and references cited therein.

13. The ordinary Evans-Polanyi relationships involve energies of activation or their analog, $\ln k$. See: H. E. O'Neal and S. W. Benson, in Free Radicals, J. K. Kochi, Ed., Vol. II, Wiley-Interscience, New York, 1973, p. 302; C. G. Screttas, J. Org. Chem., 45, 1620 (1980).

14. J. F. Garst, in Free Radicals, J. K. Kochi, Ed., WileyInterscience, New York, 1973, Vol. 1, p. 528.

Institute of Organic Chemistry

The National Hellenic Research Foundation

Athens 11635 , Greece

Received April 11, 1991

Accepted October 10, 1991

* To whom all correspondence should be addressed. 